\title{
Normalization of blood lactate as early end-point of polytrauma treatment
}

\author{
Galkova $\mathrm{K}^{1}$, Vrabelova $\mathrm{M}^{2}$ \\ Department of Anesthesiology and Intensive Medicine, Faculty Hospital of Constantine the Philosopher University, \\ Faculty of Social Sciences and Health Care, Nitra, Slovakia. galkova@gmx.net
}

\begin{abstract}
Background: The main aim of the early treatment of polytrauma is recovery of patient's physiological functions. The early achievement of therapeutic goals, mainly adequate tissue perfusion and repayment of oxygen debt, are postulated.

The aim of the study was to confirm whether blood lactate level as a quantifier of oxygen deficit, as well as normalization of blood lactate level within the first 24 hours, have an impact on the mortality and morbidity of seriously injured patients.

Methods: Sixty-nine mechanically ventilated patients with a severe trauma and organ dysfunction defined according to SOFA score and ISS $>17$, age $>15$ years, were enrolled into this retrospective study. 8 patients died within first 24 hours, 8 patients did not reach serum lactate level above $2 \mathrm{mmol} / \mathrm{l}$ on admission to hospital. The hypothesis that normalization of serum lactate level within 24 hours is related to lower mortality and morbidity, was assessed. Reduced mortality and morbidity were represented by lower severity of multi-organ dysfunction, the highest SOFA score during hospitalization, lower incidence of sepsis, number of days in ICU and artificial ventilation.

Results: The association between severity of multi-organ failure $(p=0.0006)$, mortality $(p=0.0022)$ and repayment of oxygen debt was confirmed. Hypothesis of sepsis incidence was not confirmed $(p=0.34)$. The association between number of days on artificial ventilation and number of days in ICU to repayment of oxygen debt was not confirmed either. Multivariate significant factors were age, GCS, ISS and SOFA score on patient's admission. Conclusion: The patients, who repaid oxygen debt within first 24 hours, have lower morbidity and mortality (Tab. 6, Ref. 19). Full Text in PDF www.elis.sk.

Key words: polytrauma, lactate, MOF, SOFA score.
\end{abstract}

\begin{abstract}
Abbreviations: APACHE - Acute Physiology and Chronic Health Evaluation; $\mathrm{BE}$ adm - Base Excess et admission; $\mathrm{FIO}_{2}$ - Fractional Inspired Oxygen; GCS - Glasgow Coma Scale; ICU - Intensive Care Unit; ICU days - days of ICU stay; ISS - Injury Severity Score; LACT clear - Lactate Clearance; LACT adm - serum lactate at admission; LACT 24H - serum lactate over first 24 hours; MAP - Mean Arterial Pressure; MOD - Multiple Organ Dysfunction; MOF - Multiple Organ Failure; $\mathrm{PaO}_{2}$ - arterial oxygen tension; RTS - Revised Trauma Score; SOFA - Sequential Organ Failure Assessment; TRISS - Trauma Injury Severity Score; AV days days of artificial ventilation.
\end{abstract}

Polytrauma patients have common systemic pathophysiological consequences. Hypoperfusion leads to shock with dysfunction of macro and microcirculation. The presence of oxygen debt results in an inflammatory cascade that can lead to multiorgan dysfunction. From this point of view, rapid identification and restoration

${ }^{1}$ Department of Anesthesiology and Intensive Medicine, Faculty Hospital, Faculty of Social Sciences and Health Care, Nitra, Slovakia, and ${ }^{2}$ Department of Mathematics, Faculty of Natural Sciences, University of Constantine the Philosopher in Nitra (UKF), Nitra, Slovakia

Address for correspondence: K. Galkova, MD, PhD, Department of Anesthesiology and Intensive Medicine, Faculty Hospital, Faculty of Social Sciences and Health Care, Spitalska 6, SK-949 01 Nitra 1, Slovakia. of oxygen debt are key end-points of severe trauma treatment, which can lead to better outcome of polytrauma.

Historically, we used clinical signs to identify shock: heart rate, blood pressure, urine output, which is not adequate to asses imbalance between oxygen delivery and metabolic demands of tissue, likewise cardiac output. Occult hypoperfusion can be present also in the case of normal vital signs. In the case of tissue hypoxia, lactate levels increase. Lactate as an adaptive mechanism of intermediate metabolism allows human organism to survive oxygen debt. Hyperlactatemia but better lactate clearance correlate with mortality and MOF in different types of shock $(1,2,3)$.

The aim of our study was to asses whether lactate and its normalization in blood within first 24 hours have impact on morbidity and mortality of severe trauma patients.

The management of trauma patients requires timely intervention and damage control in an attempt to achieve adequate hemodynamic parameters and systemic perfusion.

\section{Methods}

We retrospectively studied polytrauma patients at the Department of anesthesiology and intensive medicine, Faculty Hospital Nitra, who were admitted between January 1st 2008 and December 31 st 2009. During this period there were 124 polytrauma patients 
637-641

Tab. 1. The SOFA scoring system $\left(\mathrm{PaO}_{2-}\right.$ arterial oxygen tension. $\mathrm{FIO}_{2-}$ fractional inspired oxygen. $\mathrm{MAP}$ - mean arterial pressure).

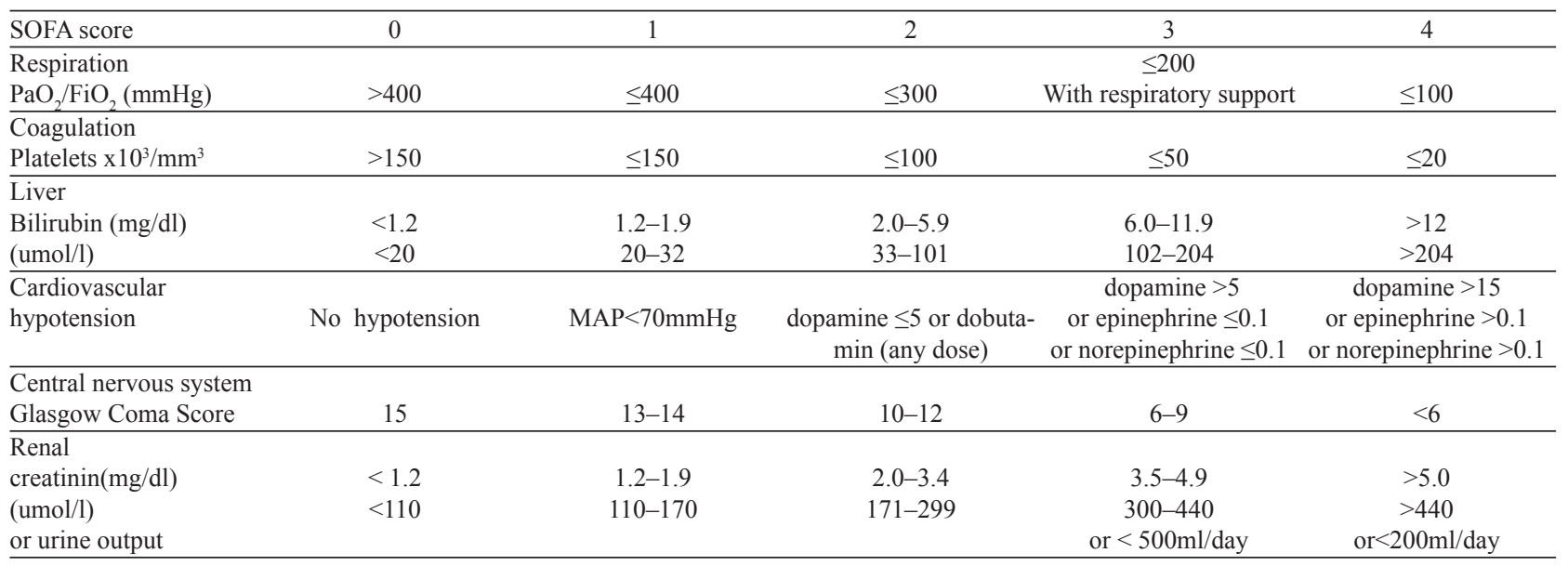

Tab. 2. Patients characteristics.

\begin{tabular}{|c|c|c|c|c|c|c|c|}
\hline \multirow[b]{2}{*}{ Variable } & \multicolumn{7}{|c|}{ Descriptive Statistics (trauma1) } \\
\hline & Valid $\mathrm{N}$ & Mean & $\begin{array}{l}\text { Confidence } \\
-95.000 \%\end{array}$ & $\begin{array}{c}\text { Confidence } \\
95.000\end{array}$ & Minimum & Maximum & Std.Dev \\
\hline age & 69 & 39.04348 & 34.42715 & 43.65981 & 16.0000 & 87.0000 & 19.21659 \\
\hline GCS & 68 & 9.26471 & 8.08881 & 10.44060 & 3.0000 & 15.0000 & 4.85804 \\
\hline ISS & 69 & 37.23188 & 33.03487 & 41.42890 & 17.0000 & 75.0000 & 17.47111 \\
\hline LACT adm & 68 & 3.81765 & 3.38145 & 4.25384 & 1.20000 & 7.80000 & 1.80207 \\
\hline LACT24h & 61 & 2.13410 & 1.85893 & 2.40927 & 0.90000 & 7.40000 & 1.07443 \\
\hline APACHE & 68 & 15.20588 & 13.43626 & 16.97550 & 0.00000 & 36.0000 & 7.31092 \\
\hline SOFA adm & 69 & 6.27536 & 5.65480 & 6.89592 & 1.00000 & 12.0000 & 2.58323 \\
\hline SOFAmax & 61 & 6.49180 & 5.69329 & 7.29031 & 1.00000 & 16.0000 & 3.11781 \\
\hline ICUdays & 69 & 15.31884 & 11.76251 & 18.87518 & 0.00000 & 60.0000 & 14.80411 \\
\hline AVdays & 69 & 10.33333 & 7.56669 & 13.09998 & 0.00000 & 45.0000 & 11.51682 \\
\hline
\end{tabular}

Tab. 3. Mann-Whitney's U Test by variable lactate clearance.

\begin{tabular}{|c|c|c|c|c|c|c|c|c|c|c|}
\hline \multirow{2}{*}{ variable } & \multicolumn{10}{|c|}{$\begin{array}{l}\text { Mann-Whitney U Test (trauma1) } \\
\text { By variable lactate clear } \\
\text { Marked tests are significant at } \mathrm{p}<.05000\end{array}$} \\
\hline & $\begin{array}{l}\text { Rank } \\
\text { Sum } \\
\text { Group } 1\end{array}$ & $\begin{array}{c}\text { Rank } \\
\text { Sum } \\
\text { Group } 2\end{array}$ & $\mathrm{U}$ & $\mathrm{Z}$ & p-level & $\begin{array}{c}\mathrm{Z} \\
\text { adjusted }\end{array}$ & p-level & $\begin{array}{l}\text { Valid N } \\
\text { Group } 1\end{array}$ & $\begin{array}{l}\text { Valid N } \\
\text { Group } 2\end{array}$ & $\begin{array}{c}2 * 1 \text { sided } \\
\text { exact } \mathrm{p}\end{array}$ \\
\hline SOFAmax & 591.000 & 840.000 & 156.000 & -3.43076 & 0.000602 & -3.45325 & 0.000554 & 29 & 24 & 0.000430 \\
\hline ICU days & 759.500 & 671.500 & 324.500 & -0.41991 & 0.6745551 & -0.42038 & 0.674210 & 29 & 24 & 0.677060 \\
\hline
\end{tabular}

admitted, and 69 of them fulfilled the inclusion criteria. Patients were eligible for inclusion when ventilated, with ISS $>17$, SOFA score $>2$. The exclusion criterion was: age below 17 . Eight patients died within first 24 hours, 8 patients did not reach serum lactate level above $2 \mathrm{mmol} / \mathrm{l}$ on admission to hospital. A decline of serum lactate level was assessed. Lactate measurements were performed using arterial blood samples by standard colorimetric laboratory method. Serum lactate level below $2 \mathrm{mmol} / \mathrm{l}$ was considered as a repayment of oxygen debt. The hypothesis, thatnormalization of serum lactate level within 24 hours is related to lower mortality and morbidity, was assessed. Reduced mortality and morbidity were represented by lower severity of multiorgan dysfunction, the highest SOFA score during hospitalization, lower incidence of sepsis, number of days in ICU and mechanical ventilation.
We collected demographic data, blood lactate levels at admission and after 24 hours, ICU days and days of artificial ventilation (AV days). Each patient was calculated for Glasgow Coma Scale (GCS), Injury Severity Score (ISS) and Acute Physiology and Chronic Evaluation (APACHE) score at admission. Severity Organ Failure Assessment (SOFA) scores were calculated at admission and then every day and a maximum during staying SOFA score was recorded (Tab. 1) and characteristics of the patients are shown on Table 2.

Used statistic methods: Mann-Whitney's U test to compare SOFA score, ICU stay, AV days in two groups categorized according to normalization of blood lactate up to 24 hours. Statistical significance was accepted to respond to p. Fisher's exact test was used on categorical data to asses the correlation of sepsis and 
Tab. 4.Fisher's exact test of dependency of mortality and lactate normalization.

\begin{tabular}{lccc}
\hline & \multicolumn{3}{c}{$2 \times 2$ tab (lact.clear-exitus) } \\
\cline { 2 - 4 } & Column 1 & Column 2 & Row Totals \\
\hline Frequencies. row 1 & 17 & 7 & 24 \\
Percentage of & $\mathbf{3 2 . 0 7 5 \%}$ & $\mathbf{1 3 . 2 0 8}$ & $\mathbf{4 5 . 2 8 3 \%}$ \\
Frequencie. row 2 & 29 & 0 & 29 \\
Percentage of & $\mathbf{5 4 . 7 1 7 \%}$ & $\mathbf{0 . 0 0 0 \%}$ & $\mathbf{5 4 . 7 1 7 \%}$ \\
Column totals & 46 & 7 & 53 \\
Percentage of & $\mathbf{8 6 . 7 9 2 \%}$ & $\mathbf{1 3 . 2 0 8 \%}$ & \\
Chi-square (df $=1$ ) & 9.75 & $\mathrm{p}=.0018$ & \\
V-square (df =1) & 9.56 & $\mathrm{p}=.0020$ & \\
Yates corrected Chi-square & 7.37 & $\mathrm{p}=.0066$ & \\
Phi-square & 18388 & & \\
Fisher exact p.one -tailed & 7.37 & $\mathrm{p}=.0022$ & \\
Two-tailed & & $\mathrm{p}=.0022$ & \\
McNemarChi-square(A/D) & 15.06 & $\mathrm{p}=.0001$ & \\
Chi/square (B/C) & 12.25 & $\mathrm{P}=.0005$ & \\
\hline
\end{tabular}

surviving with early normalization of lactate within first 24 hours. Multivariate analysis of covariants was done.

\section{Results}

The association between severity of multi-organ failure and normalization of lactate level $<2 \mathrm{mmol} / 1$ in the first 24 hours of polytrauma patients was confirmed by the Mann-Whitney's rank-sum test. The level of statistical significance was $\mathrm{p}<0.05$ (Tab. 3).

Significant correlation between days of ICU stays and days on artificial ventilation was not confirmed. Dependency of mortality and lactate normalization was confirmed with Fisher's exact test $(\mathrm{p}=0.0022)$ (Tab. 4).

Hypothesis of the incidence of sepsis related to lactate normalization was not confirmed by Fisher's exact test ( $p=0.34$ ) (Tab. 5).

Multivariate significant factors were age, ISS and SOFA score on patient's admission. On the contrary, serum lactate at admission, base excess and number of transfusions over the first 24 hours were not multivariable significant (Tab. 6).

\section{Discussion}

Though management of severe trauma during last period has changed, morbidity and mortality is still high. The mechanisms underlying morbidity and mortality in trauma patients are divided into (1) direct trauma associated alterations (e.g., organ injury, hemorrhage, and/or severe traumatic brain injury) and (2) secondary or delayed alterations, such as overwhelming inflammatory reactions, deterioration of microcirculation, and multiple organ failure (MOF). Currently, MOF is thought to be responsible for up to $80 \%$ of all deaths in the intensive care unit (4). Multiple organ dysfunction is known to be the leading cause of mortality in the patients survived the initial hours after trauma (5).

The early achievement of therapeutic goals, mainly early restoration of perfusion and repayment of oxygen debt are crucial to prevent MOF, which is the main cause of morbidity and mortality not only in polytrauma, but also in other critically ill patients.
Tab. 5. Fisher's exact test of incidence of sepsis related to lactate normalization.

\begin{tabular}{lccc}
\hline & \multicolumn{3}{c}{$2 \times 2$ tab (lact.clear-sepsa) } \\
\cline { 2 - 4 } & Column 1 & Column 2 & Row Totals \\
\hline Frequencies. row 1 & 11 & 13 & 24 \\
Percentage of & $\mathbf{2 0 . 7 5 5 \%}$ & $\mathbf{2 4 . 5 2 8 \%}$ & $\mathbf{4 5 . 2 8 3 \%}$ \\
Frequencie. row 2 & 16 & 13 & 29 \\
Percentage of & $\mathbf{3 0 . 1 8 9 \%}$ & $\mathbf{2 4 . 5 2 8 \%}$ & $\mathbf{5 4 . 7 1 7 \%}$ \\
Column totals & 27 & 26 & 53 \\
Percentage of & $\mathbf{5 0 . 9 4 3 \%}$ & $\mathbf{4 9 . 0 5 7 \%}$ & \\
Chi-square (df =1) & 46 & $\mathrm{p}=.4984$ & \\
V-square (df =1) & 45 & $\mathrm{p}=.5025$ & \\
Yates corrected Chi-square & 16 & $\mathrm{p}=.6884$ & \\
Phi-square & 00865 & & \\
Fisher exact p.one -tailed & & $\mathrm{p}=.3444$ & \\
Two-tailed & & $\mathrm{p}=.5857$ & \\
McNemarChi-square(A/D) & 04 & $\mathrm{p}=.8383$ & \\
Chi/square (B/C) & 14 & $\mathrm{p}=.7103$ & \\
\hline
\end{tabular}

Tab. 6. Multivariate tests.

\begin{tabular}{|c|c|c|c|c|c|c|}
\hline & Effect & Value & $\mathrm{F}$ & $\begin{array}{c}\text { Hypothesis } \\
\text { df }\end{array}$ & $\begin{array}{c}\text { Error } \\
\text { df }\end{array}$ & Sig \\
\hline \multirow[t]{4}{*}{ Intercept } & Trac & .018 & $.261^{\mathrm{a}}$ & 3.000 & 42.000 & .853 \\
\hline & Wilk's Lambda & .982 & $.261^{\mathrm{a}}$ & 3.000 & 42.000 & .853 \\
\hline & Hotelling's Trace & .019 & $.261^{\mathrm{a}}$ & 3.000 & 42.000 & .853 \\
\hline & Roy's Largest & .019 & $.261^{\mathrm{a}}$ & 3.000 & 42.000 & .853 \\
\hline \multirow[t]{4}{*}{$\overline{\text { Age }}$} & Pillai's Tra & .318 & $6.524^{\mathrm{a}}$ & 3.000 & 42.000 & .001 \\
\hline & Wilk's Lar & .682 & $6.524^{\mathrm{a}}$ & 3.000 & 42.000 & .001 \\
\hline & Hotelling's & .466 & $6.524^{\mathrm{a}}$ & 3.000 & 42.000 & .001 \\
\hline & Roy's Larg & .466 & $6.524^{\mathrm{a}}$ & 3.000 & 42.000 & .001 \\
\hline \multirow[t]{4}{*}{$\overline{\mathrm{GCS}}$} & Pillai's Trace & .243 & $4.499^{\mathrm{a}}$ & 3.000 & 42.000 & .008 \\
\hline & Wilk's Lambda & .757 & $4.499^{\mathrm{a}}$ & 3.000 & 42.000 & .008 \\
\hline & Hotelling's & .321 & $4.499^{\mathrm{a}}$ & 3.000 & 42.000 & .008 \\
\hline & Roy's Largest & .321 & $4.499^{\mathrm{a}}$ & 00 & 42.000 & .008 \\
\hline \multirow[t]{4}{*}{$\overline{\mathrm{ISS}}$} & Pillai's Tra & .185 & $3.172^{\mathrm{a}}$ & 00 & 42.000 & .034 \\
\hline & Wilk' & .815 & $3.172^{\mathrm{a}}$ & & & .034 \\
\hline & Hotelling's & .227 & $3.172^{\mathrm{a}}$ & 00 & 42.000 & .034 \\
\hline & Roy's Largest Root & .227 & $3.172^{\mathrm{a}}$ & 3.000 & 42.000 & .034 \\
\hline \multirow[t]{4}{*}{ Transf } & Pillai's Tra & .138 & $2.240^{\mathrm{a}}$ & 3.000 & 42.000 & .098 \\
\hline & Wilk's Lai & .862 & $2.240^{\mathrm{a}}$ & .000 & 42.000 & .098 \\
\hline & Hot & .160 & $2.240^{\mathrm{a}}$ & & 42.000 & .098 \\
\hline & Roy & .160 & $2.240^{\mathrm{a}}$ & 00 & 42.000 & .098 \\
\hline \multirow[t]{4}{*}{ Lakta-p } & Pillai's Tr & .033 & $.471^{\mathrm{a}}$ & 3.000 & 42.000 & .704 \\
\hline & Wilk's Lambda & .967 & $.471^{\mathrm{a}}$ & 00 & 42.000 & .704 \\
\hline & Hot & .034 & $.471^{\mathrm{a}}$ & & 42.000 & .704 \\
\hline & Roy's Largest Root & .034 & $.471^{\mathrm{a}}$ & 00 & 42.000 & .704 \\
\hline \multirow[t]{4}{*}{$\overline{\mathrm{BE} \_ \text {prij }}$} & Pillai's Tra & .143 & $2.330^{\mathrm{a}}$ & 3.000 & 42.000 & .088 \\
\hline & Wilk & .857 & $2.330^{\mathrm{a}}$ & 3.000 & 42.000 & .088 \\
\hline & Hotelling's & .166 & $2.330^{\mathrm{a}}$ & 3.000 & 42.000 & .088 \\
\hline & Roy's Largest Root & .166 & $2.330^{\mathrm{a}}$ & 3.000 & 42.000 & .088 \\
\hline \multirow[t]{4}{*}{ SOFA_p } & Pillai's Trace & .382 & $8.664^{\mathrm{a}}$ & 3.000 & 42.000 & .000 \\
\hline & Wilk & .618 & $8.664^{\mathrm{a}}$ & & 42.000 & .000 \\
\hline & Hotelling's Trace & .619 & $8.664^{\mathrm{a}}$ & 3.000 & 42.000 & .000 \\
\hline & Roy's Largest Root & 619 & $8.664^{\mathrm{a}}$ & 3.000 & 42.000 & .000 \\
\hline \multirow[t]{4}{*}{ Lact_clear } & Pillai's Trace & .228 & $4.130^{\mathrm{a}}$ & 3.000 & 42.000 & .012 \\
\hline & Wilk's Lambda & .772 & $4.130^{\mathrm{a}}$ & 3.000 & 42.000 & .012 \\
\hline & Hotelling's Trac & .295 & $4.130^{\mathrm{a}}$ & 3.000 & 42.000 & .012 \\
\hline & Roy's Largest Root & .295 & $4.130^{\mathrm{a}}$ & 3.000 & 42.000 & .012 \\
\hline
\end{tabular}

${ }^{a}$ Exact statistic ${ }^{b}$ Design: intercept + age + GCS +ISS + transf + lacta_prij + BE_prij + SOFA_p +Lact_clear

Resuscitation end-points allow the physician to rapidly identify a perturbation between oxygen delivery and consumption. End-points allow uniformity to assess the adequacy of resuscita- 
$637-641$

tion: preventing under- and over resuscitation and act as a basis to compare outcome measures in resuscitation trials.

As in other studies, we used blood lactate level as metabolic correlate of oxygen debt and stress metabolism together with effective lactate clearance as therapeutic end-point. Blood lactate levels are closely related to outcome in critically ill patient $(1,2$, $3,6,7,8,9)$. We also showed that early normalization of lactate level is significantly connected with lower morbidity and mortality in polytrauma.

Consistently with previous literature $(10,11)$, we defined organ dysfunction according to the SOFA score definitions. There are three classes of scoring systems available in the evaluation of trauma patients in the intensive care unit (ICU): those based on physiological parameters (APACHE score), Simplified Acute Physiology Score, and Revised Trauma Score (RTS)], those based on definition of the entity and localization of trauma (ISS), and those considering either anatomical lesions or deranged physiological parameters - Trauma Injury Severity Score (TRISS).

The main limitations of these scoring systems in evaluating critically ill patients are: some authors have emphasized that the prognostic value of the available scoring systems is strictly dependent on treatment. Two patients with a similar severity of illness might have greatly differing scores based on appropriate or inadequate resuscitation. The prognosis assessment based on scores is only applicable to a study population, not to an individual patient. The components of some scores are not easily obtained. An ideal prognostic index should be based on variables available by standard routine examinations, applicable to heterogeneous patient groups, readily and widely measurable, and not related to personal judgments or affected by the treatment. Majority of current severity scoring is based on an estimation of mortality. A treatment that does not affect mortality may still be effective: if morbidity rather than mortality is considered as the major outcome measure, the impact of intensive care on quality of life, length of stay in the ICU, costs, etc. can be evaluated. SOFA score is composed of scores from six organ systems (respiratory, cardiovascular, hepatic, coagulation, renal, and neurological) graded from 0 to 4 according to the degree of dysfunction/failure. The SOFA score can reliably describe organ dysfunction in trauma patients. Regular and repeated scoring may be helpful in identifying categories of patients at major risk of prolonged ICU stay or death.

SOFA score better correlates with physiological changes and the therapeutic response than anatomical scores. SOFA score is common for septic patients but lots of authors use it for scoring of MOF in trauma patients over time $(10,11)$. SOFA score quantifies and describes the evolution of organ dysfunction over time (12, $13)$, and has been validated in trauma patients. The incidence of MOF after injury in recent studies differs between 5-25\% (10). In Ulvik study (14) half of patients had MOF. Differences in ICU admission policies and case-mix may complicate direct comparison with other studies. In addition, the application of different scoring systems for assessment of MOF makes direct comparison difficult. In our hospital, trauma patients without severe organ failure are usually treated outside the ICU.
To assess the degree severity of MOF we use the highest number during hospitalization in ICU. In our study significant association between normalization of lactate within first 24 hours and severity of MOF was confirmed by the Mann-Whitney's ranksum test. Multivariate significant factors were GCS, ISS and also SOFA score at admission. Also Antonelli showed that surviving patients had lower SOFA score at admission as patients who died (15). The dependency of mortality onthe early normalization of blood lactate was confirmed by Fisher's exact test.

There is a therapeutic window when hypoxic cells are able to revert to normal state without damage. This hypothesis was confirmed also by Shoemaker (16) with supra-normal hemodynamic support, because his interventions were tight with surgical insult. Also Rivers (17) with septic patients $\mathrm{ScvO} 2$ changes and lactate in interval a few hours was effective in time.

We have to understand lactate as an adaptation to an anaerobic condition, but also other processes may induces high serum lactate, like stimulation of aerobic glycolysis by catecholamines in stress metabolism, which helps organism to survive (18). But our intervention must be in the window of time while metabolic failure is not present. The time to intervention becomes relevant: early and effective treatment may allow the cell to revert to a normal state, as long as the oxygen machinery is intact. Once the mitochondria are deranged, energy failure occurs even in the presence of normoxia. The lactate increase in critically ill patients may therefore be viewed as an early marker of a potentially reversible state (19).

\section{Conclusion}

Early normalization of blood lactate level is related to low grade of MOF and lower mortality of seriously injured patients. Targeted resuscitation strategy after severe trauma should comprise of early identification of oxygen debt and use of resuscitation end point - blood lactate level to achieve better outcomes of patients.

\section{References}

1. Jeng JC, Jablonski K, Bridgeman A, Jordan MH. Serum lactate, not base deficit, rapidly predicts survival after major burns. Burns 2002; 28 (2): 161-166.

2. Husain FA, Martin MJ, Mullenix PS et al. Serum lactate and base deficit as predictors of mortality and morbidity. Am J Surg 2003; 185 (5): 485-491.

3. McNelis J, Marini CP, Jurkiewicz A et al. Prolonged lactate clearance is associated with increased mortality in the surgical intensive care unit. Am J Surg 2001; 182 (3): 481-485.

4. Baue AE. Let's be splitters, rather than Lumpers. Crit Care Med 2004; 32 (6): 1440-1441.

5. Maier B, Lefering R, Lehnert M et al. Early versus late onset of multiple organ failure is associated with differing patterns of plasma cytokine after severe trauma. Shock 2007; 28 (6): 668-674. 
6. Meregalli A, Oliviera RP, Friedman G. Occult hypoperfusion is associated with increased mortality in hemodynamically stable, high-risk, surgical patients. Critical Care 2004; 8 (2): 654-667.

7. Crowl AC, Young JS, Kahler DM et al. Occult hypoperfusion is associated with increased morbidity in patients undergoing early femur fracture fixation. J Trauma 2000; 48 (2): 260-267.

8. Claridge JA, Crabtree TD, Pelletier SJ et al. Persistent occult hypoperfusion is associated with a significant increase in infection rate and mortality in major trauma patients. J Trauma 2000; 48 (1): 8-14.

9. Ciesla DJ, Moore EE, Johnson JL et al. A 12-year prospective study of postinjury multiple organ failure: has anything changed? Arch Surg 2005; 140 (4): 432-440.

10. Jhanji S, Lee C, Watson D et al. Microvascular flow and tissue oxygenation after major abdominal surgery: association with post-operative complications. Intensive Care Med 2009; 35 (4): 671-677.

11. Durham RM, Moran JJ, Mazuski JE et al. Multiple organ failure in trauma patients. J Trauma 2003; 55 (5): 608-616.

12. Vincent JL, Demendonca A, Cantraine F et al. Use of the SOFA score to assess the incidence of organ dysfunction/failure in intensive care units: results of a multicentric, prospective study. Crit Care Med 1998; 26 (10): 1793-1800.
13. Vincent JL, Sakr Y, Sprung CL et al. Sepsis in European intensive care units: results of the SOAP study. Crit Care Med 2006; 37 (2): 344-353.

14. Ulvik A, Wentzel-Larsen T, Flaatten H. Trauma patients in the intensive care unit: short- and long-term survival and predictors of 30-day mortality. Acta Anaesthesiol Scand 2007; 51 (1): s. 171-177.

15. Antonelli M, Moreno R, Vincent JL et al. Application of SOFA score to trauma patients, Intensive Care Med 1999; 25 (2): 389-394

16. Shoemaker WC, Paul L, Appel MP et al. Prospective Trial of Supranormal Values of Survivors as Therapeutic Goals in High- Risk Surgical Patients, Chest 2009; 94 (6): 1176-1186.

17. Rivers E, Nguyen B, Havstad S et al. Early goal-directed therapy in the treatment of severe sepsis and septic shock. N Engl J Med 2001; 345 (12): $1368-1377$.

18. Levy B, Gibot S, Franck P, Cravoisy A, Bollaert PE. Relation between muscle $\mathrm{Na}+\mathrm{K}+$ ATPase activity and raised lactate concentrations in septic shock: a prospective study. Lancet 2005; 365 (9462): 871-875.

19. Valenza F, Aletti G, Fossali $\mathbf{T}$ et al. Lactate as a marker of energy failure in critically ill patients: hypothesis. Crit Care 2005; 9 (2): 588-593.

Received December 2, 2011 Accepted August 10, 2012. 